\title{
GPPS-BJ-2019-0007
}

\section{PARAMETER INVESTIGATION OF RING MICROPHONE ARRAY FOR ROTATING SOUND SOURCE LOCALIZATION}

\author{
Wei ZHOU \\ Engineering Research Center of Gas Turbine \\ and Civil Aero Engine, Ministry of Education; \\ School of Mechanical Engineering, \\ Shanghai Jiao Tong University \\ ZW_SJTU@sjtu.edu.cn \\ Shanghai 200240, China
}

\author{
Mingsui YANG \\ AECC Shenyang Engine Research Institute \\ yangmingsui@126.com \\ Shenyang, Liaoning 110015, China
}

\author{
Huan BAO \\ Engineering Research Center of Gas Turbine \\ and Civil Aero Engine, Ministry of Education; \\ School of Mechanical Engineering, \\ Shanghai Jiao Tong University \\ 1170209103860817@sjtu.edu.cn \\ Shanghai 200240, China
}

\author{
Wei MA \\ Engineering Research Center of Gas Turbine \\ and Civil Aero Engine, Ministry of Education; \\ School of Aeronautics and Astronautics, \\ Shanghai Jiao Tong University \\ mawei@sjtu.edu.cn \\ Shanghai 200240, China
}

\begin{abstract}
As society developed expeditiously, more attentions have been paid towards harm of noise. Hence, noise reduction has become a significant in industries. Localization of rotating sound source plays a main role in the process of noise reduction. Ring microphone array is essential in order to localize the rotating sound source using frequency domain algorithm, since it's able to construct a virtual array rotating synchronously with the rotating sound source in order to remove Doppler Effect. Array radius and the number of microphones play their key roles in ring microphone array, which they will significantly affect the measurement accuracy and costs. In spite of that, there is no evidence proof that radius and microphone number have an impact on the accuracy of rotating sound source localization. The intention for this study is to qualitatively analyze the impacts of radius and microphone number on the measurement accuracy. The intensity, position, resolution and dynamic range of the initial sound source are regarded as evaluation indexes by using rotational beamforming, deconvolution algorithms to compare the results. As conclusion, only when the ratio of the array radius to the rotating radius of the sound source is nearly 1 and the ratio of microphone number to the circumference of the ring array within $128 / 0.5 \pi \sim 32 / 0.5 \pi$, rotating beamforming would have a better spatial resolution and dynamic range. Moreover, the intensity error obtained by deconvolution algorithm is only $0.0128 \mathrm{~dB}$, and the position deviation has no
\end{abstract}

business with array parameters. In a nutshell, this study provided guidance in localization of rotating sound source using ring microphone array, where it kept a balance between measurement accuracy and cost needed.

Keywords: Microphone array; Rotating sound; Source localization; Array radius; Microphone number

\section{INTRODUCTION}

A microphone array has been widely used to study the problem of sound source localization. The processing of noise reduction has become necessary in industry, because of the higher and higher requirements for the living environment. The following noise reduction design depends on how to locate the sound source better. In practical engineering, most of the noise were generated by rotating mechanical motion, such as open rotor engine blade rotation in aero-engine field ${ }^{1}$, Wind turbine blade rotation in civil field ${ }^{2,3}$, and so on. Therefore, the localization of the rotating sound source is very important in the noise reduction design for the rotating noise.

A virtual array synchronized with the rotating sound source can be constructed by a ring microphone array ${ }^{4}$, so that the sound source and the virtual array are relatively stationary. It is widely used in the rotating sound source localization, because it can eliminate the Doppler effect caused by the motion of the sound source 5 . The algorithm precision optimization and array parameter optimization both can locate the sound source accurately and efficiently. 
In the development of algorithm optimization, researchers have successively designed a simple and reliable delay-and-sum beamforming algorithm (DAS), which has the disadvantage of low spatial resolution. Then the deconvolution algorithms, such as DAMAS and CLEAN-SC with high spatial resolution and clean image have been designed ${ }^{6-8}$. On the other hand, the installation parameters of the microphone array, such as the number of microphones and the array radius have an effect on the actual measurement process ${ }^{9,10}$. For the measured sound source, the measurement results will get higher precision with more microphones. However, the cost of the array measurement will increase because of the high cost of microphones, so it is not desirable in the actual measurement process. Meanwhile, the size of the array radius will also affect the accuracy of the measurement results. At present, a qualitative study on the influence of array installation parameters in the process of rotating sound source localization has not been carried out by engineering and academic circles.

In this paper, the influences of microphone array parameters on the rotating sound source localization were studied systematically mainly from the aspects of microphone number and array radius. The numerical analysis method was adopted in this paper in order to avoid the error effect analysis introduced by the experiment method. This paper used beamforming algorithm and deconvolution algorithms to compare, in order to obtain accurate data results. The effects of microphone number and array radius on the simulation results were studied respectively. The variation of the beamforming resolution and dynamic range was analyzed by calculating the bandwidth of the $-3 \mathrm{~dB}$ main lobe and the sidelobe suppression level of beamforming. The variation curves of the localization position deviation and the localization intensity error were given by comparing the deconvolution results with the position and intensity of the initial sound source.

The full paper is divided into four parts. In the second section, the frequency domain algorithms of ring microphone array are introduced, including rotating beamforming algorithm and deconvolution algorithms. In the third section, the simulation models are designed and the parameters of the simulation models are given. In the fourth section, the simulation results are compared with the position and intensity of the initial sound source, and the deviation curves of the localization position and the localization intensity are given. In the fifth section, the simulation results are analyzed and discussed, the following research will be introduced. In the sixth section, the research conclusions and some suggestions for practical engineering measurements are given. The purpose of this paper is to study the influence of array parameters on the localization of rotating sound source and to guide the practical application in order to achieve the measurement goal of accurate measurement and cost-saving.

\section{METHODOLOGY}

\section{Beamforming Algorithm}

Considering a grid of $\mathrm{N}$ focus points $x_{t}$, the frequency domain formulation for the basic delay-and-sum beamformer is

$$
\mathrm{B}\left(x_{t}\right)=W_{\Omega}^{H}\left(x_{t}\right) \operatorname{CSM} W_{\Omega}\left(x_{t}\right), t=1,2 \ldots(1)
$$

with the superscript $H$ denoting conjugate transposition, $\Omega$ is the angular frequency of rotating sound source, and the dimension of guide vector $W$ corresponds to the number of microphones used, including phase shift and amplitude correction ${ }^{11-13}$.

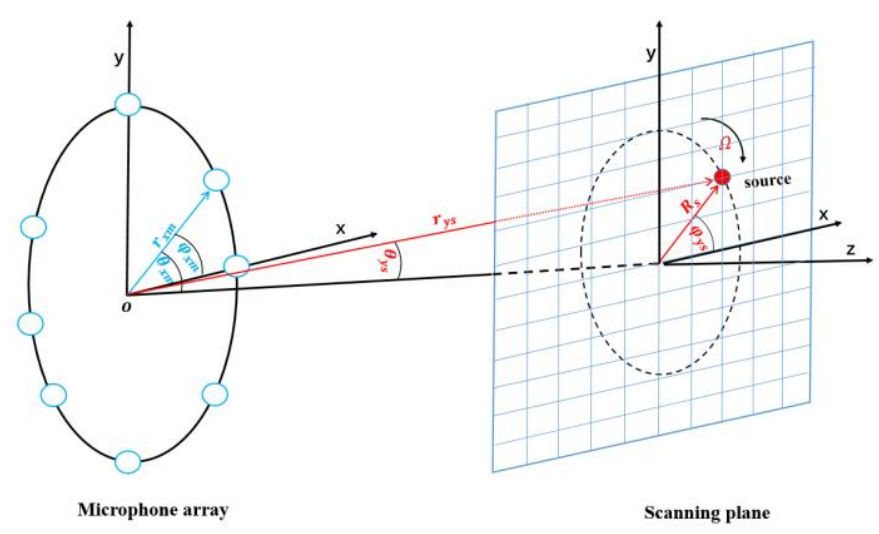

Figure 1 Schematic Diagram

Figure 1 shows the grid coordinates and microphone array coordinates under spherical coordinates respectively. grid coordinates $\mathbf{y}(\mathbf{s})=\left(r_{y_{s}}, \theta_{y_{s}}, \varphi_{y_{s}}\right)$ microphone array coordinates $\mathbf{x}(\mathbf{m})=\left(r_{x m}, \theta_{x m}, \varphi_{x m}\right)$

$$
\begin{gathered}
G_{\Omega}(s, m)=-i \sum_{m_{0}=-\infty}^{+\infty} e^{i m_{0}\left(\varphi_{x m}-\varphi_{y s}\right)} k m_{0} \sum_{n=\left|m_{0}\right|}^{+\infty} N_{m_{0} n}^{2} b_{n}\left(k_{m_{0}}, r_{x m}, r_{y s}\right) \\
\cdot P_{n}^{m_{0}}\left(\cos \theta_{x m}\right) P_{n}^{m_{0}}\left(\cos \theta_{y s}\right)
\end{gathered}
$$

With the normalization factor

$$
N_{m_{0} n}=\sqrt{\frac{2 n+1}{4 \pi} \frac{\left(n-m_{0}\right) !}{\left(n+m_{0}\right) !}}
$$

and frequency shift wave number due to rotation

$$
k_{m_{0}}=\frac{\omega+m_{0} \Omega}{c}
$$

and $P_{n}^{m_{0}}(\cdot)$ is first kind of Legendre function of degree $n$ and order $m_{0}$, and

$$
b_{n}= \begin{cases}j_{n}\left(k_{m_{0}} r_{y}\right) h_{n}^{\left(\delta_{m_{0}}\right)}\left(k_{m_{0}} r_{x}\right) & r_{y} \leq r_{x} \\ j_{n}\left(k_{m_{0}} r_{y}\right) h_{n}^{\left(\delta_{m_{0}}\right)}\left(k_{m_{0}} r_{y}\right) & r_{y}>r_{x}\end{cases}
$$

Where $j_{n}(\cdot)$ is the first kind of spherical Bessel function, and $h_{n}^{\left(\delta_{m_{0}}\right)}(\cdot)$ is $\delta_{m_{0}}$ kind of spherical Hankel function and

$$
\delta_{m_{0}}= \begin{cases}2 & k_{m 0} \geq 0 \\ 1 & k_{m 0}<0\end{cases}
$$

the Green's function $G$ is introduced 


$$
\begin{gathered}
G_{\Omega}=\left[\begin{array}{llllc}
G_{\Omega}^{1} & \cdots & G_{\Omega}^{S} & \cdots & G_{\Omega}^{S}
\end{array}\right] \\
=\left[\begin{array}{ccccc}
G_{\Omega}(1,1) & \cdots & G_{\Omega}(1, s) & \cdots & G_{\Omega}(1, S) \\
\vdots & & \vdots & & \vdots \\
G_{\Omega}(m, 1) & \cdots & G_{\Omega}(m, s) & \cdots & G_{\Omega}(m, S) \\
\vdots & & \vdots & & \vdots \\
G_{\Omega}(M, 1) & \cdots & G_{\Omega}(M, s) & \cdots & G_{\Omega}(M, S)
\end{array}\right]
\end{gathered}
$$

Calculate the guide vector matrix $W$

$$
W_{\Omega}=\left[W_{\Omega}^{1} \cdots \mathrm{W}_{\Omega}^{S} \cdots W_{\Omega}^{S}\right] W_{\Omega}^{S}=\frac{G_{\Omega}^{S}}{\left\|G_{\Omega}^{S}\right\|_{2}^{2}}
$$

$\boldsymbol{C S M}$ in formula (1) is expressed as the cross-spectral matrix. These are time signals measured by microphones and calculated by Welch spectral estimation method ${ }^{14}$. The principal diagonal of matrix $\mathrm{C}$ can be excluded from the analysis so as to reduce the error caused by the irrelevant background noise produced by the measurement, because the self-spectrum does not contain the phase difference between signals or the obvious amplitude information in other matrix terms ${ }^{15}$.

The beamformer output $\mathrm{B}\left(x_{t}\right)$ can be interpreted as the actual sound source distribution derived from the convolution of a point spread function. The properties of the beamforming algorithm and the selection of the geometry of the microphone array introduce the systematic error, which lead to a rather fuzzy distribution map of the sound source. Therefore, Many high resolution algorithms have been proposed, such as DAMAS CLEAN-SC.

\section{Deconvolution Algorithms}

DAMAS algorithm is an iterative algebraic deconvolution method. The deconvolution acoustic power $q_{w}^{\prime}$ is obtained via point spread function matrix $\mathrm{A}$ and beamforming results. The computational grid of DAMAS needs to be fine enough, and too thick grid points will also cause spatial mixing of sound source distribution. In addition, DAMAS has the disadvantages of large computation and long operation time ${ }^{16}$. Some studies have shown that DAMAS with low frequency resolution has some advantages for the larger aperture array ${ }^{17}$. Ma \& Liu ${ }^{7}$ proposed a method to compress the number of grids by dropping invalid grids, thus solving the problem of computing efficiency. CLEAN-SC, as one of the most efficient algorithms at present, its basic principle is to search for the maximum peak of beamforming output and copy its value into a clean mapping graph. Then, identify the coherent parts and subtract them from the original dirty graph, leaving different maximum values. Repeat this process until the more important source is not found in the original dirty graph or the maximum number of iterations is reached ${ }^{18}$. In this paper, the two algorithms are used to compare and analyze the results.

\section{Resolution and Dynamic range}

The beamforming resolution and dynamic range in this paper are calculated by constructing the point spread function matrix A

$$
\begin{gathered}
\mathrm{A}=\left[\begin{array}{ccccc}
p s f(y(1) / y(1)) & \cdots & p s f(y(1) / y(s)) & \cdots & p s f(y(1) / y(S)) \\
\vdots & & \vdots & & \vdots \\
p s f(y(s) / y(1)) & \cdots & p s f(y(s) / y(s)) & \cdots & p s f(y(s) / y(S)) \\
\vdots & & \vdots & \vdots \\
p s f(y(S) / y(1)) & \cdots & p s f(y(S) / y(s)) & \cdots & p s y(y(S) / y(S))
\end{array}\right] \\
\operatorname{psf}(\mathrm{y}(\mathrm{v}) / \mathrm{y}(\mathrm{s}))=\left|\left(W_{\Omega}^{v}\right)^{H} G_{\Omega}^{S}\right|^{2}, \mathrm{v}=1,2,3, \ldots, \mathrm{S}, \mathrm{s}=1,2,3, \ldots, \mathrm{S} \quad(10) \\
A=\left(\left(W_{\Omega}\right)^{\mathrm{H}} G_{\Omega}\right)^{2}
\end{gathered}
$$

After constructing the point spread function, selecting the point spread function at the center of the grid to analyze the dynamic range and resolution. the highest peak point in the two-dimensional beamforming diagram is placed in the center of the image, and the horizontal line of the peak point is the $\mathrm{X}$ axis. The spectral bandwidth at peak point $-3 \mathrm{~dB}$ is the beamforming resolution. he dynamic range of beamforming is determined by calculating the vertical displacement between the peak and the secondary peak ${ }^{19}$.

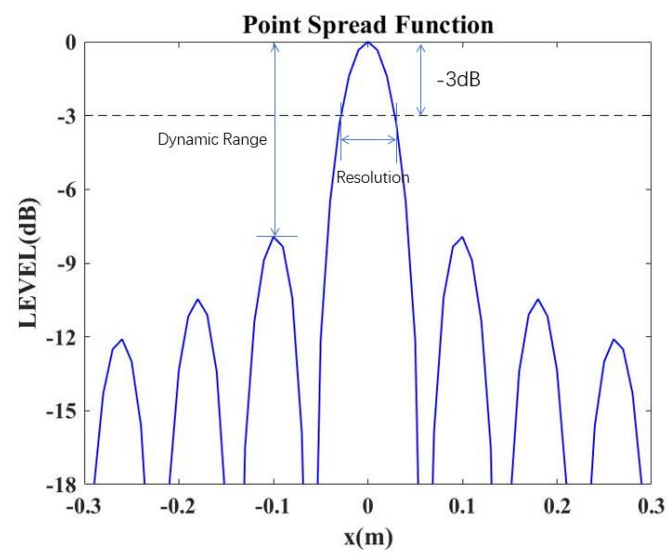

Figure 2 PSF Results

\section{APPLICATION SIMULATIONS}

In this paper, the influences of microphone array parameters on the sound source position and sound source intensity are analyzed, and simulation models are designed. Figure 3 shows the source distribution

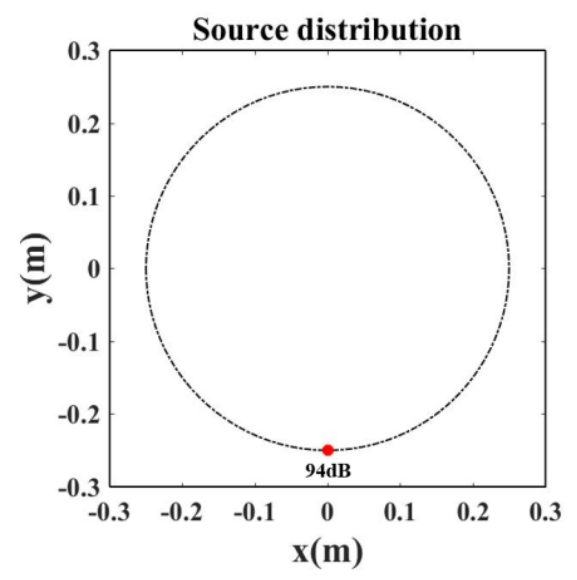

Figure 3 Source Distribution

Two sets of simulation models are designed aiming at the influences of array radius and microphone number. Sound source processing parameters and simulation models are as follows 
Table 1 Data Processing Parameters

Sound Source Type

Sound Velocity

Sound Rotating Radius

Sound Rotating Speed

Array Shape

Number of Grids

Initial Position

Measured Distance Turn

Sampling Frequency

Sampling Period

CLEAN-SC Iterations

DAMAS Iterations

The first set of simplified simulation model used only a single point source. The frequency of the sound source was $1,600 \mathrm{~Hz}$ at low frequency, $5,000 \mathrm{~Hz}$ at intermediate frequency and $10,000 \mathrm{~Hz}$ at high frequency. Three typical sound sources frequency have been chosen, then changed the radius of the array from $0.125 \mathrm{~m} \sim 1.0 \mathrm{~m}$ respectively, set the number of microphones to 128 , with the sound source parameters kept unchanged. The relationship between the array radius and the sound source rotation radius was explored by calculating the beamforming resolution and dynamic range, which minimizes the position deviation and the intensity error.

The second group explored the effects of microphone number and followed the sound source model in the second set. Choosing the frequency of the three sound sources as above, keeping the position of the sound source, rotating radius and rotating speed unchanged, changing the number of microphones from 16 128 respectively, the simulations were carried out at the array radius $0.25 \mathrm{~m}$. This group aimed to find the ratio of microphone number to radius which satisfies the minimum position deviation and intensity error obtained by deconvolution calculation, while the relationship between microphone number and array perimeter on beamforming resolution and dynamic range were observed at the same time.

\section{RESULTS AND DISCUSSION}

\section{Results}

\section{Effects caused by array radius}

Figure 4 shows the intensity errors when the array radius is changed. The vertical coordinate indicates the absolute value of the deviation between the simulation intensity and the initial intensity of the sound source, and the transverse coordinate indicates the ratio of the array radius to the sound source rotating radius. The sound source rotating radius is $0.25 \mathrm{~m}$ in this process, and the number of microphones used is 128. The radius of the array is $0.125 \mathrm{~m}, 0.25 \mathrm{~m}, 0.5 \mathrm{~m}, 0.75 \mathrm{~m}$, $1.0 \mathrm{~m}$ respectively. In comparison with Figure 4 (a) and Figure 4 (b), we can find that under this array condition, the accuracy of sound source intensity using CLEAN-SC algorithm is higher than that of DAMAS algorithm. In Figure 4 (a), the accuracy of the measurement intensity is higher when the radius of the array is close to the rotation radius of the sound source, and the error range increases obviously with the increase of the radius ratio. In Figure 4 (b), the DAMAS algorithm has a large deviation when the frequency of the sound source is $1,600 \mathrm{~Hz}$ and the array radius is smaller than the rotating radius.

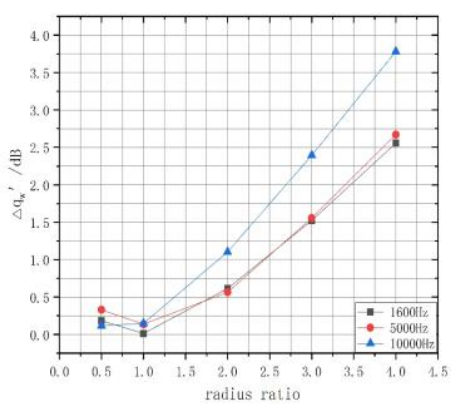

(a) CLEAN-SC Results

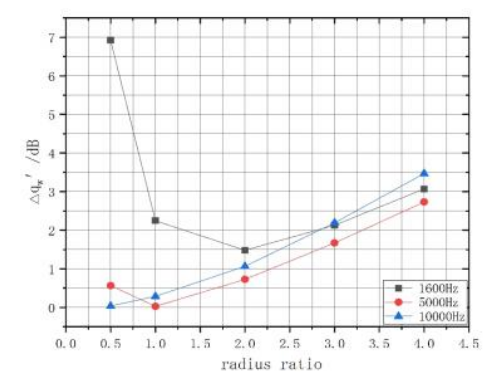

(b) DAMAS Results

Figure $\mathbf{4}$ Localization Intensity Errors

In Figure 5, the cloud images of three frequency sources at the radius of $0.125 \mathrm{~m}, 0.25 \mathrm{~m}$ and $1.0 \mathrm{~m}$ are given respectively.

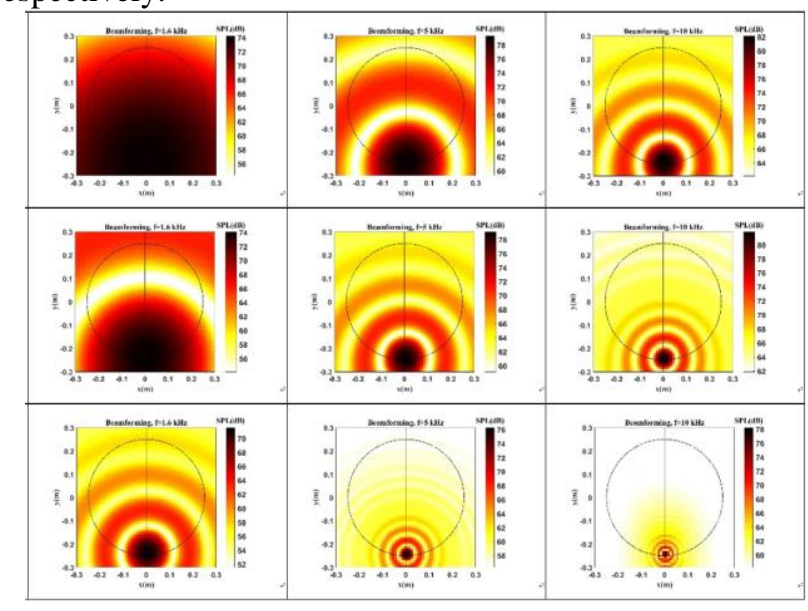

Figure 5 Localization Positions

Considering the influence of the array radius on the position error of the sound source, the maximum position $\mathrm{y}^{\prime}\left(s_{0}\right)$ of the sound source is obtained from $\mathrm{q}_{\mathrm{w}}^{\prime}$, and the position error of the source location is calculated $\Delta \mathrm{d}=$ $\left|y\left(s_{0}\right)-y^{\prime}\left(s_{0}\right)\right|$. Figure 6 is the relationship curve of position deviation between radius ratio, in which the position deviation range is $0 \mathrm{~m} \sim 0.01 \mathrm{~m}$. With the increase of the ratio of array radius to sound source rotating radius, the position deviation 
of sound source remains unchanged. It is shown that changing the array radius does not cause the position deviation of rotating sound source without any deviation in array installation. The accuracies of DAMAS and CLEAN-SC algorithms are also verified.

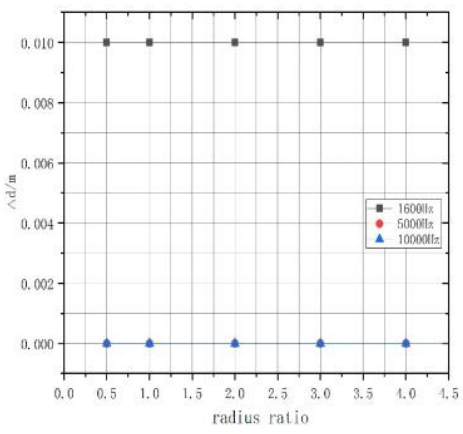

\section{Figure 6 Localization Position Deviations}

Figures 7-8. indicate relationship curves of beamforming resolution and dynamic range with array radius are given respectively. The beamforming resolution is obtained by calculating the bandwidth at $-3 \mathrm{~dB}$ of the highest peak value. The sidelobe suppression of the maximum point to the secondary peak position is dynamic range, and the point spread function matrix PSF is used to calculate the beamforming resolution. It can be found that the highest resolution is $0.6 \mathrm{~m}$ in Figure 7, when the resolution is large, the acoustic source point will be located coherently. Therefore, the lower the resolution is, the clearer the distribution of the sound source will be. The resolution becomes better with the increase of the size of the array. In the case of low frequency $1,600 \mathrm{~Hz}$, the resolution value is larger when the array size is small, and the suppression effect for sidelobe is poor, so there will be aliasing dirty source. It has the best resolution when the array radius is larger. Figure 8 shows the relationship between the dynamic range and the array radius. The sound source resolution in the low frequency stage is very poor, so the dynamic range can be approximately considered to be infinity, but it does not mean that it is an excellent dynamic range. The dynamic range indicates suppression effect of the main lobe to the side lobe. In practical localization, the maximum sound pressure point should be determined as the sound source location, and the side lobe should be well suppressed.

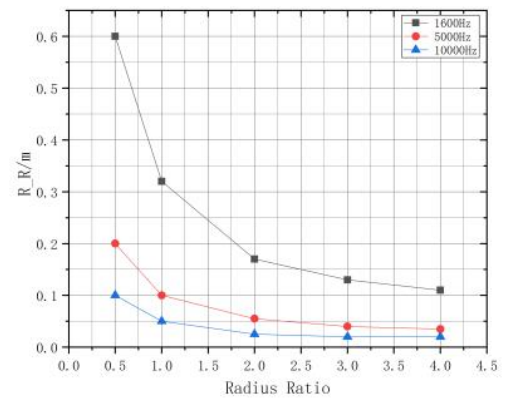

Figure 7 Resolution Ranges

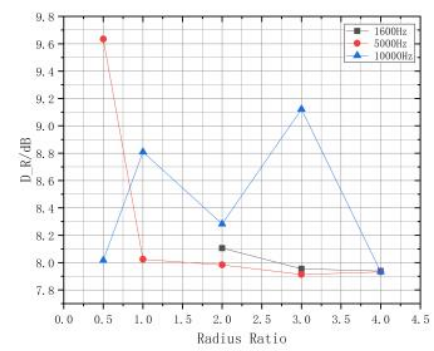

Figure 8 Dynamic Ranges

\section{Effects caused by microphone number}

Figure 9 shows the intensity errors when microphone number is changed. In the condition of CLEAN-SC algorithm, it is not difficult to find that the stability of the CLEAN-SC algorithm in the middle and low frequencies is good. The error range of the sound source intensity is $0.0129 \mathrm{~dB} \sim 4.8279 \mathrm{~dB}$, and the intensity error increases with the decrease of the ratio of number to radius and the deviation value in the highfrequency phase is more obvious with the decrease of the number of the microphones, and the deviation is large.

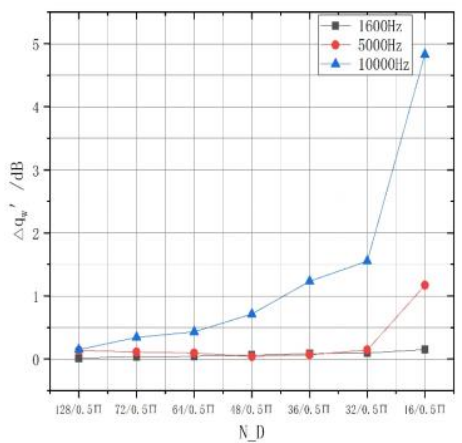

(a) CLEAN-SC Results

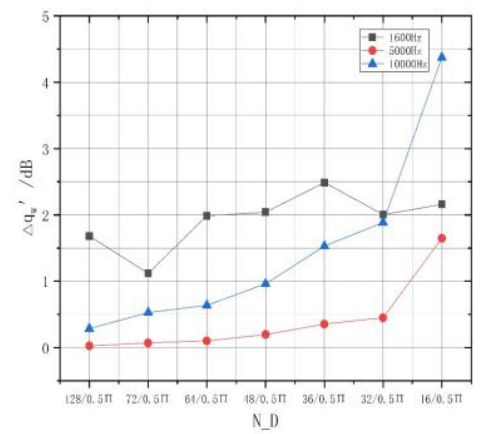

(b) DAMAS Results

Figure 9 Localization Intensity Errors

In the case of DAMAS algorithm, it can be seen that when the DMAS algorithm is employed at low frequency, the intensity fluctuation is large, and when the center frequency is high, the error increases with the microphone number 
decreases. The minimum localization intensity error of DAMAS algorithm is about $0.0246 \mathrm{~dB}$ and the maximum positioning intensity error is about $4.3745 \mathrm{~dB}$ when N_D ratio is $128 / 0.5 \pi \sim 32 / 0.5 \pi$. DAMAS algorithm is good at the intermediate frequency, but there is a significant deviation when the microphone number is 16 .

Figure 10 shows the location cloud of the three frequency sources at the number of microphones at 16,64 and128.

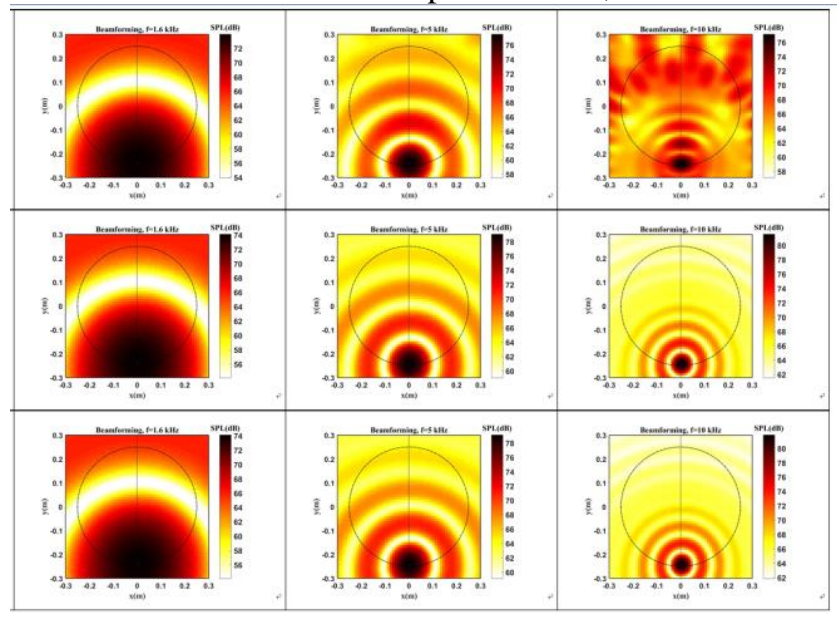

Figure 10 Localization Positions

In Figure 11, the deviation of the localization position is basically unchanged, with the decrease of the microphone number to array radius, indicating that in the case of the array installation is correct, the change of the array N_D ratio has no effect on the deviation of the localization position. The deviation of low-frequency position value may be caused by the serious aliasing of low-frequency sound source and poor resolution, so it is impossible to locate the position of sound source accurately. At high frequency, there is a large deviation at a small N_D ratio, which indicates that the size of the subarray may not be accurately measured when the number of microphones is reduced to 16 .

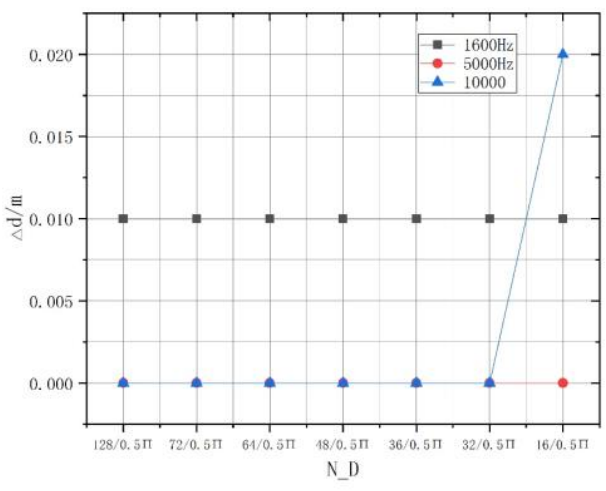

Figure 11 Localization position deviations

Figures 12-13 indicate the beamforming resolution and dynamic range. According to the change curve of resolution and the influence of array radius, it can be found that the size of resolution is independent of the number of microphones, but only related to the change of array radius, and increases with the size of the array. It is also found in the above image that the resolution value decreases gradually with the increase of frequency, and the resolution is good, but the resolution of aliasing is poor in the low frequency stage. When the dynamic range is observed, the low frequency range is considered to be infinite, so the resolution of the low frequency segment is poor without sidelobe suppression. The number of middle and high frequency microphones varies little, fluctuates up and down, and there is no obvious phenomenon, but it is mainly related to frequency.

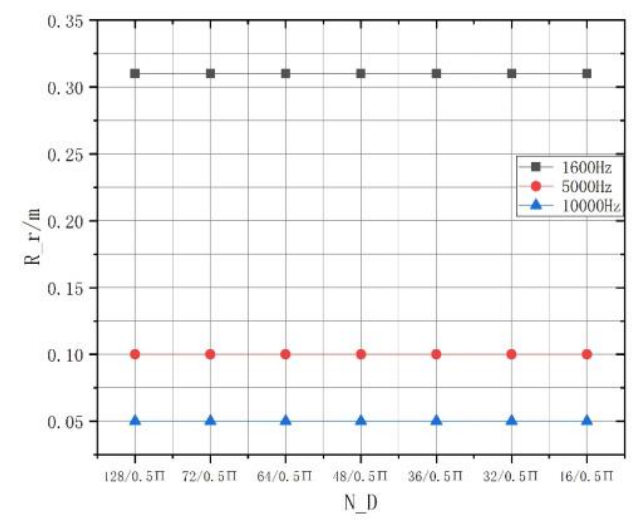

Figure 12 Resolutions Ranges

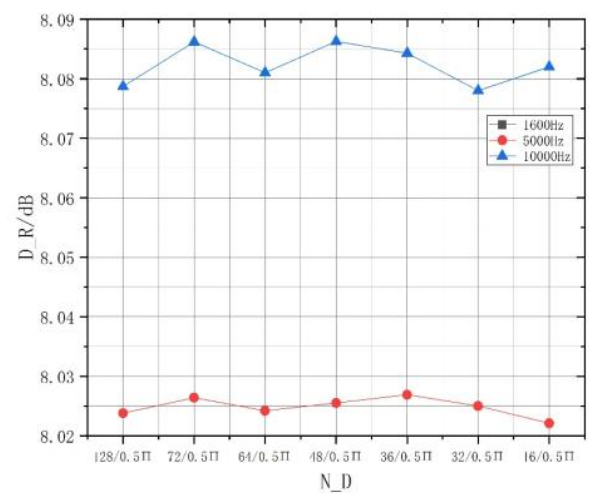

Figure 13 Dynamic Ranges

\section{Discussion}

The influences of the installation parameters of the microphone array on the localization of the rotating sound source are explored in this paper. The variations of the localization intensity and the position are mainly studied, and the beamforming resolution and dynamic range are also included in the performance inspection index. This paper aims to find the appropriate array installation parameters for the academic and engineering community. Then analyse the effects of changes on the array radius and microphone number in the localization of the sound source, so as to provide the theoretical guidance for the microphone array measurements in the practical engineering. All relevant issues will be discussed in this section.

The primary task of sound source localization is measuring intensity and position accurately. It can be found 
out that the variation trend of intensity error is obvious when the array size changes. However, when considering the influences of microphone number change on localization, only $0.25 \mathrm{~m}$ array radius was selected, and five groups of microphone number change experiments were carried out. Then, the influences of microphone number change on different array sizes will be investigated.

Secondly, the performance of the sound source localization process can be evaluated by resolution and dynamic range. Since the grid point used in this paper was $61 \times 61$, there may be some errors in the data acquisition, so we can increase the number of grid points to explore the changing rule of resolution and dynamic range for further.

Finally, the simulation models in this paper are based on the localization distance of $1 \mathrm{~m}$. The following simulation can explore the influences of localization distance changes in the localization process. The linkage effect between the location distance, the microphone number and array radius for rotating sound sources can be studied.

\section{CONCLUSION}

In this paper, the influences of the localization of the sound source caused by the changes of the array parameters are studied, and conclusions are drawn as follows.

1) No matter how the installation parameters of the microphone array are changed, the deviation of the position of the sound source is within the allowed range, if there is no deviation of the installation angle. It indicates that the position of the sound source will not be affected by changing the parameters of the array. It also reflects the accuracy of CLEAN-SC and DAMAS deconvolution algorithms.

2) When the Radius Ratio is 1 , the intensity error is only $0.0128 \mathrm{~dB}$, and the intensity error increases linearly with the increase of the Radius Ratio. It is indicated that the array radius should be as close as possible to the sound source rotating radius in the measurement. When the radius of the fixed array is large, the intensity error increases gradually with the microphone number decreases, and there is a great difference in the error as for the high frequency range. At the same time, it is found that the precision of CLEAN-SC algorithm is higher than that of DAMAS algorithm.

3) Resolution and dynamic range are used to evaluate the performance of microphone array. The value of resolution and dynamic range determines the position of the identified sound source and the accuracy of the intensity. The resolution value decreases with the array size increases. The larger the array radius is, the better the resolution is. When the array radius is constant and the number of microphones is changed, the dynamic range has little effect on the resolution and dynamic range.

4) In the practical engineering survey, the array radius should equal to the rotating radius, the microphone number should within the N_D 128/0.5 $\pi \sim 32 / 0.5 \pi$. Under this arrangement, the intensity and position of sound source, and dynamic range of beamforming resolution are better.

\section{NOMENCLATURE}

$\begin{array}{ll}\Omega & \text { Angular Frequency } \\ W & \text { Guide Vector } \\ G & \text { Green's Function } \\ \text { CSM } & \text { Cross Spectral Matrix } \\ \text { PSF } & \text { Point Spread Function } \\ \text { FFT } & \text { Fast Fourier Transform } \\ q_{w}^{\prime} & \text { Deconvolution Acoustic Power } \\ \Delta q_{w}^{\prime} & \text { Localization Intensity Error } \\ \Delta \mathrm{d} & \text { Localization Position Error } \\ \text { Radius Ratio } & \text { Array Radius to Rotating Radius } \\ \text { N_D } & \text { Microphone Number to Array Diameter } \\ \text { D_R } & \text { Dynamic Range } \\ \text { R_R } & \text { Resolution Range }\end{array}$

\section{ACKNOWLEDGMENTS}

This work was supported by the Special Funds for Basic Scientific Research of Central Universities of China.

\section{REFERENCES}

[1] Dürrwächter L., et al. (2019). Numerical assessment of open-rotor noise shielding with a coupled approach. AIAA Journal: 1-11. DOI: 10.2514/1.J057531.

[2] Fleig O., and Arakawa C. (2004). Numerical simulation of wind turbine tip noise. 42nd AIAA Aerospace Sciences Meeting and Exhibit. DOI: 10.2514/6.2004-1190.

[3] Pourazarm P., et al. (2014). Flow-induced instability of wind turbine blades. 32nd ASME Wind Energy Symposium. DOI: $10.2514 / 6.2014-1219$.

[4] Herold G., and Sarradj E. (2015). Microphone array method for the characterization of rotating sound sources in axial fans. Noise Control Engineering Journal, 63(6), pp. 546551. DOI: 10.3397/1/376348.

[5] Li Z.Y., and Duraiswami R. (2007). Flexible and optimal design of spherical microphone arrays for beamforming. IEEE Transactions on Audio, Speech and Language Processing, 15(2), 10.1109/TASL.2006.876764

$\mathrm{pp}$. 702-714.DOI:

[6] Zeng Y., and Hendriks R. C. (2014). Distributed delay and sum beamformer for speech enhancement via randomized gossip. IEEE/ACM Transactions on Audio, Speech, and LanguageProcessing22(1), pp. 260-273. DOI:10.1109/TASLP.2013.2290861.

[7] Ma W., and Liu X. (2017). DAMAS with compression computational grid for acoustic source mapping. Journal of Sound and Vibration 410, pp. 473-484. DOI: 10.1016/j.jsv.2017.03.027.

[8] Chu Z.G., et al. (2019). Deconvolution using CLEAN-SC for acoustic source identification with spherical microphone arrays. Journal of Sound and Vibration 440, pp. 161-173. DOI: 10.1016/j.jsv.2018.10.030.

[9] Carballo-Crespo A., and Takeda K. (2009). An investigation of microphone array installation effects. 47th AIAA Aerospace Sciences Meeting including The New Horizons Forum and Aerospace Exposition. DOI: 10.2514/6.2009-883.

[10] Brandstein M.S., et al. (1996). A localization-error-based method for microphone-array design. 1996 IEEE International Conference on Acoustics, Speech, and Signal Processing 
Conference Proceedings, Atlanta, GA, USA, 1996, pp. 901904 vol. 2.DOI: 0-7803-3 192-3/96.

[11] Arcondoulis E.J.G., and Liu Y. (2018) Acoustic beamforming array design using an iterative microphone removal method. 2018 AIAA/CEAS Aeroacoustics Conference. DOI: 10.2514/6.2018-2807.

[12] Dougherty R.P., et al. (2014). Functional beamforming for aeroacoustic source distributions. 20th AIAA/CEAS Aeroacoustics Conference. DOI: 10.2514/6.2014-3066.

[13] Merino-Martínez R., et al. (2016). Functional beamforming applied to imaging of flyover noise on landing aircraft. Journal of Aircraft 53(6), pp. 1830-1843. DOI: 10.2514/1.C033691.

[14] Kay S. M. (1987). Modern Spectral Estimation: Theory \& Application. NJ, USA: Englewood Cliffs Publishing.

[15] Ocker C., and Pannert W. (2017). Calculation of the cross spectral matrix with Daniell's method and application to acoustical beamforming. Applied Acoustics 120, pp. 59-69. DOI: 10.1016/j.apacoust.2017.01.011.

[16] Lylloff O., et al. (2015). Improving the efficiency of deconvolution algorithms for sound source localization. Journal of Acoustical Society of America 138(1), pp. 172180.DOI: 10.1121/1.4922516.

[17] Sarradj E., et al. (2018). Array methods: Which one is the best. 7th Berlin Beamforming Conference 2018.BeBeC-2018S01.

[18] Fleury V., and Bulté J. (2011). Extension of deconvolution algorithms for the mapping of moving acoustic sources. Journal of Acoustical Society of America 129(3), pp. 1417-1428. DOI: 10.1121/1.3531939.

[19] Hald J., et al. (2012). High-resolution fly-over beamforming using a small practical array. 18th AIAA/CEAS Aeroacoustics Conference (33rd AIAA Aeroacoustics Conference). DOI: 10.2514/6.2012-2229. 